\title{
Is X-linked Recessive Ichthyosis a High Risk for Basal Cell Carcinoma?
}

\author{
Keiko Wakumoto,* Yuichi Yoshida $\uparrow$ and Osamu Yamamoto $\uparrow$ \\ *Division of Dermatology, Matsue Red Cross Hospital, Matsue 690-0886, Japan, and †Division of Dermatology, Department of \\ Medicine of Sensory and Motor Organs, School of Medicine, Tottori University Faculty of Medicine, Yonago 683-8503, Japan
}

\begin{abstract}
We report a case of $\mathrm{X}$-linked recessive ichthyosis with basal cell carcinoma. An 86-year-old man was referred to our department for evaluation of a 1-year history of an ulcer on his left leg. He had suffered from dry skin with scales on his entire body since childhood. Histopathological examination of the lower leg revealed intradermal proliferation of a solid nest of basaloid cells showing unclear palisading in the periphery. Marked hyperkeratosis and hypergranulosis were also seen in the adjacent dermis. Loss of the steroid sulfatase gene was found on chromosome X. Based on these finding, we made a diagnosis of $\mathrm{X}$-linked recessive ichthyosis with basal cell carcinoma. To our knowledge, there has been no report of X-linked recessive ichthyosis with cutaneous malignancy. We discuss the risk of skin cancer in relation to ichthyosis.
\end{abstract}

Key words basal cell carcinoma; inherited ichthyosis; $\mathrm{X}$-linked recessive ichthyosis

Congenital ichthyoses are genodermatoses caused by mutations in the genes responsible for keratinocytic differentiation and skin barrier function. ${ }^{1}$ There have been sporadic case reports of patients with various types of ichthyoses who developed skin cancers. ${ }^{2-5}$ However, there has been no report of $\mathrm{X}$-linked recessive ichthyosis with cutaneous malignancy. We report a rare case of $\mathrm{X}$-linked recessive ichthyosis with basal cell carcinoma and discuss the risk of skin cancers in relation to ichthyosis.

\section{PATIENT REPORT}

An 86-year-old man was referred to us due to a 1-year history of a gradually enlarging ulcer on his left leg. Physical examination revealed an ulcerated nodule, $4 \times 2.5 \mathrm{~cm}$ in diameter, on the left lower leg (Fig. 1a). He had suffered from dry skin with scales on his entire body since childhood (Fig. 1b), but there was no family

Corresponding author: Keiko Wakumoto, $\mathrm{MD}, \mathrm{PhD}$

w_hikaru0622@yahoo.co.jp

Received 2019 December 20

Accepted 2020 January 27

Online published 2020 February 20

Abbreviations: BCC, basal cell carcinoma; SCC, squamous cell carcinoma history of ichthyosis. Histopathological examination of a biopsy from the lower leg revealed intradermal proliferation of a solid nest of basaloid cells showing unclear palisading in the periphery. Marked hyperkeratosis and partial hypergranulosis were also seen in the adjacent epidermis (Figs. 1c and d). Loss of the steroid sulfatase gene was found on chromosome X in FISH (fluorescence in situ hybridization) (Fig. 1e). Based on these findings, we made a diagnosis of $\mathrm{X}$-linked recessive ichthyosis with basal cell carcinoma (BCC).

\section{DISCUSSION}

$\mathrm{X}$-linked recessive ichthyosis is a rare form of inherited ichthyosis, which is caused by mutations in the steroid sulfatase gene. There are some case reports suggesting an increased risk of skin cancers in patients with various forms of congenital ichthyosis. Natsuga et $\mathrm{al}^{2}$ reviewed a case series with skin malignancies in inherited ichthyoses, including 12 cases of keratitisichthyosis-deafness syndrome, 7 cases of autosomal recessive congenital ichthyosis, 3 cases of Netherton syndrome and 6 cases of miscellaneous ichthyosis. Reported skin malignancies included squamous cell carcinoma (SCC), BCC, malignant proliferating trichilemmal tumor, melanoma, malignant fibrous histiocytoma and cutaneous lymphoma. Among those tumors, the most frequent tumor was SCC ( 24 out of 28 tumors). The authors pointed out that the age at onset of the first skin malignancy ranged from 15 to 54 years and recommended routine surveillance of skin cancers. Recently, a case of SCC (in a 51-year old patient) and a case of melanoma (in a 54-year old patient) in association with ichthyosis vulgaris at relatively young ages have been reported. ${ }^{3,4}$ A particular severe subtype of ichthyosis seems to be prone to skin cancers. However, there has been no report on the occurrence of skin cancers in patients with X-linked recessive ichthyosis. These differences might be the differences in explained by causative genetic defects in ichthyosis subtypes. In addition, other factors such as scaling with chronic inflammation might be related to skin carcinogenesis, but the skin barrier function of patients with X-linked recessive ichthyosis is minimally affected, resulting in milder skin abnormalities. In general, the average age at onset of BCC in healthy individuals was 70.7 years in 

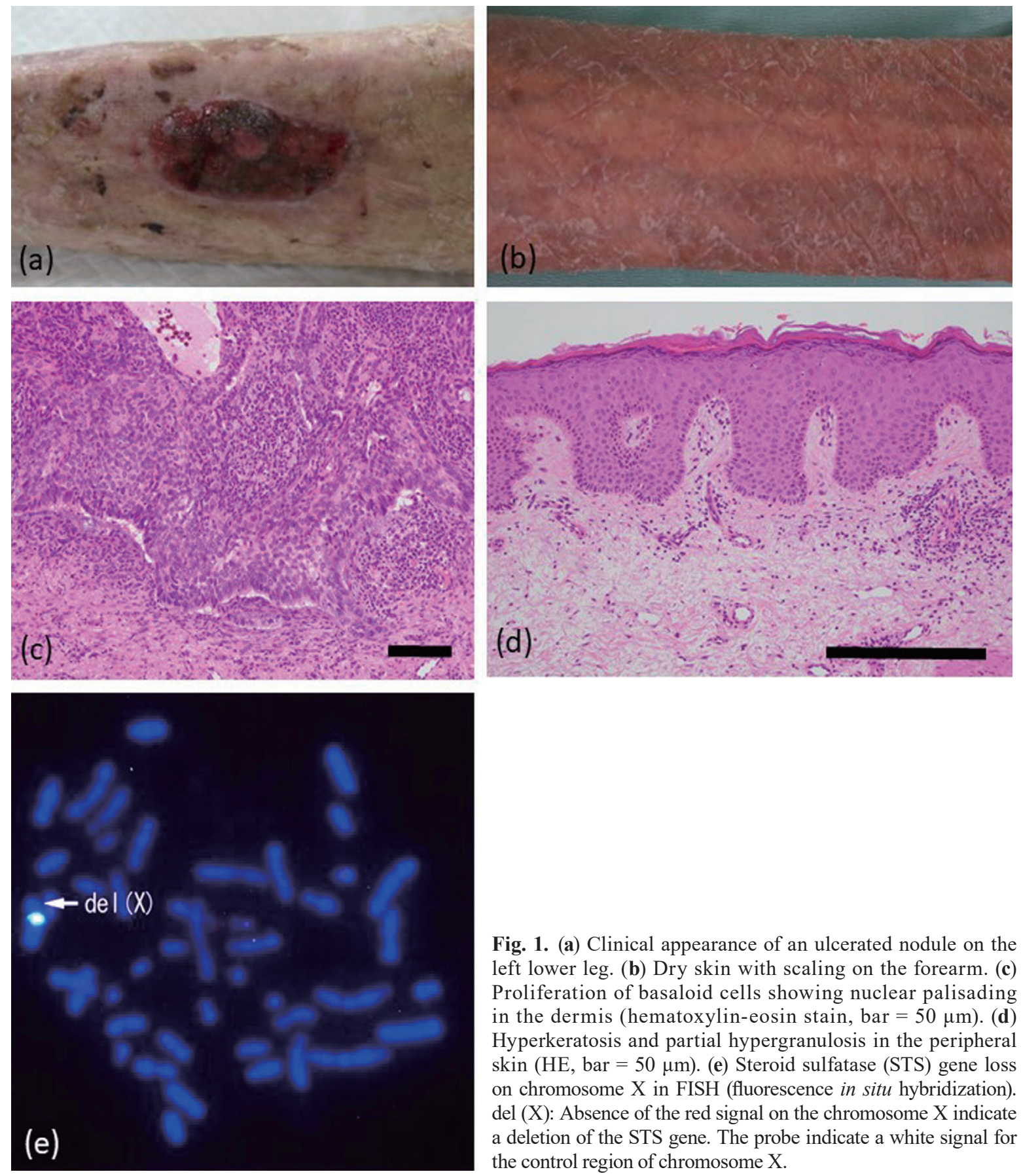

Fig. 1. (a) Clinical appearance of an ulcerated nodule on the left lower leg. (b) Dry skin with scaling on the forearm. (c) Proliferation of basaloid cells showing nuclear palisading in the dermis (hematoxylin-eosin stain, bar $=50 \mu \mathrm{m}$ ). (d) Hyperkeratosis and partial hypergranulosis in the peripheral skin $(\mathrm{HE}$, bar $=50 \mu \mathrm{m})$. (e) Steroid sulfatase (STS) gene loss on chromosome $\mathrm{X}$ in FISH (fluorescence in situ hybridization). del (X): Absence of the red signal on the chromosome $\mathrm{X}$ indicate a deletion of the STS gene. The probe indicate a white signal for the control region of chromosome X.

Japan. ${ }^{6}$ Our patient developed BCC at 86 years of age. Ghosh et al. ${ }^{7}$ reported X-linked recessive ichthyosis is the second most common form of congenital ichthyosis. Although there are some reports of skin cancers in other ichthyoses, including the most common form (ichthyosis vulgaris) or a rare form (such as KID syndrome), there has been no report on the occurrence of skin cancers in patients with X-linked recessive ichthyosis. Therefore, we consider that $\mathrm{BCC}$ is probably coincidental and that the risk of skin cancers is not high, especially in patients with X-linked recessive ichthyosis. Therefore, it is most likely that routine surveillance of skin cancers is unnecessary for patients with X-linked recessive ichthyosis.

\section{The authors declare no conflict of interest.}

\section{REFERRENCES}

1 Takeichi T, Akiyama M. Inherited ichthyosis: non-syndromic forms. J Dermatol. 2016;43:242-51. DOI: 10.1111/13468138.13243, PMID: 26945532 
2 Natsuga K, Akiyama M, Shimizu H. Malignant skin tumours in patients with inherited ichthyosis. Br J Dermatol. 2011;165:263-8. DOI: 10.1111/j.1365-2133.2011.10381.x PMID: 21517795

3 Cerra-Franco A, Grethlein SJ, Bertrand TE, Wooden WA, Saito NG. Congenital ichthyosis patient with squamous cell carcinoma of the skin who received concurrent chemoradiation: A case report. Adv Radiat Oncol. 2018;3:76-80. DOI: 10.1016/j.adro.2017.09.006, PMID: 29556584

4 Tas F, Erturk K, Demirkesen C, Goktay F. Cutaneous melanoma in association with ichthyosis vulgaris. J Pediatr Hematol Oncol. 2017;39:630. DOI: 10.1097/MPH.0000000000000953, PMID: 28902080
5 Elbaum DJ, Kurz G, MacDuff M. Increased incidence of cutaneous carcinomas in patients with congenital ichthyosis. J Am Acad Dermatol. 1995;33:884-6. DOI: 10.1016/01909622(95)90428-X, PMID: 7593802

6 Takenouchi T, Takatsuka S. Long-term prognosis after surgical excision of basal cell carcinoma: A single institutional study in Japan. J Dermatol. 2013;40:696-9. DOI: 10.1111/13468138.12207, PMID: 23803167

7 Ghosh A, Ahar R, Chatterjee G, Sharma N, Jadhav SA. Clinico-epidermiological study of congenital ichthyosis in a tertiary care center of Eastern India. Indian J Dermatol. 2017;62:606-11. PMID: 29263534 